\title{
Plasma cleaning reliability over pressure and power ranges
}

Cameron Moore

Vacuum Sciences, Prescott, Arizona, United States

The use of oxygen-containing plasmas to clean and decontaminate vacuum systems of hydrocarbons and silicones is established technology. Despite broad acceptance of this approach, technological hurdles remain:

(1) Pressure in the plasma is a key parameter that controls reach and breadth of the cleaning volume. For SEMs the preferred pressures are in the range of 1-3 Pa (7 to $20 \mathrm{mTorr}$ ), and this range can be problematic as it make plasma ignition more difficult (due to higher required breakdown voltages as described by the Paschen criteria). Decreasing pressure presents a reliability issue as plasma ignition failure becomes more likely (or requires technical stop-gap measures to prevent).

(2) Varying the plasma pressure causes difficulties because the size of plasma sheaths will vary, in turn causing the matching properties of RF-excited plasmas to shift, resulting in a loss of power coupling from RF amplifier to plasma. Changing pressure presents a performance degradation as plasma efficiency to generate cleaning species uncontrollably changes.

At M\&M-2019 we introduced our PM100 plasma cleaning system (see Figure 1) that represents a major technological upgrade in both design and operation, in addition to addressing these hurdles [1]. As described at that time, the PM100 is a fully integrated (single enclosure) design that operates at powers as low as 1 Watt and pressures < $1 \mathrm{~Pa}$ (5 mTorr).

In this paper we will present two sets of data that address our progress. An example of reliable ignition is shown in Figure 2, where plasma ignition at 20 mTorr (10 seconds on, 10 seconds off) is demonstrated 25 times sequentially. Here it can be observed that the plasma ignites to a repeatable level as based on photodiode measurement of plasma optical emission, that the DC power draw is constant (at appr. 60\% efficiency), and that expected gradual heating of the RF power amplifier occurs and stabilizes after a few minutes.

Additional data will be shown that, due to proprietary design features, the PM100 can be operated from 5 to 100 mTorr with little change in plasma impedance. This stability in RF matching properties allows pressure variation with minimal changes in cleaning species generation. 


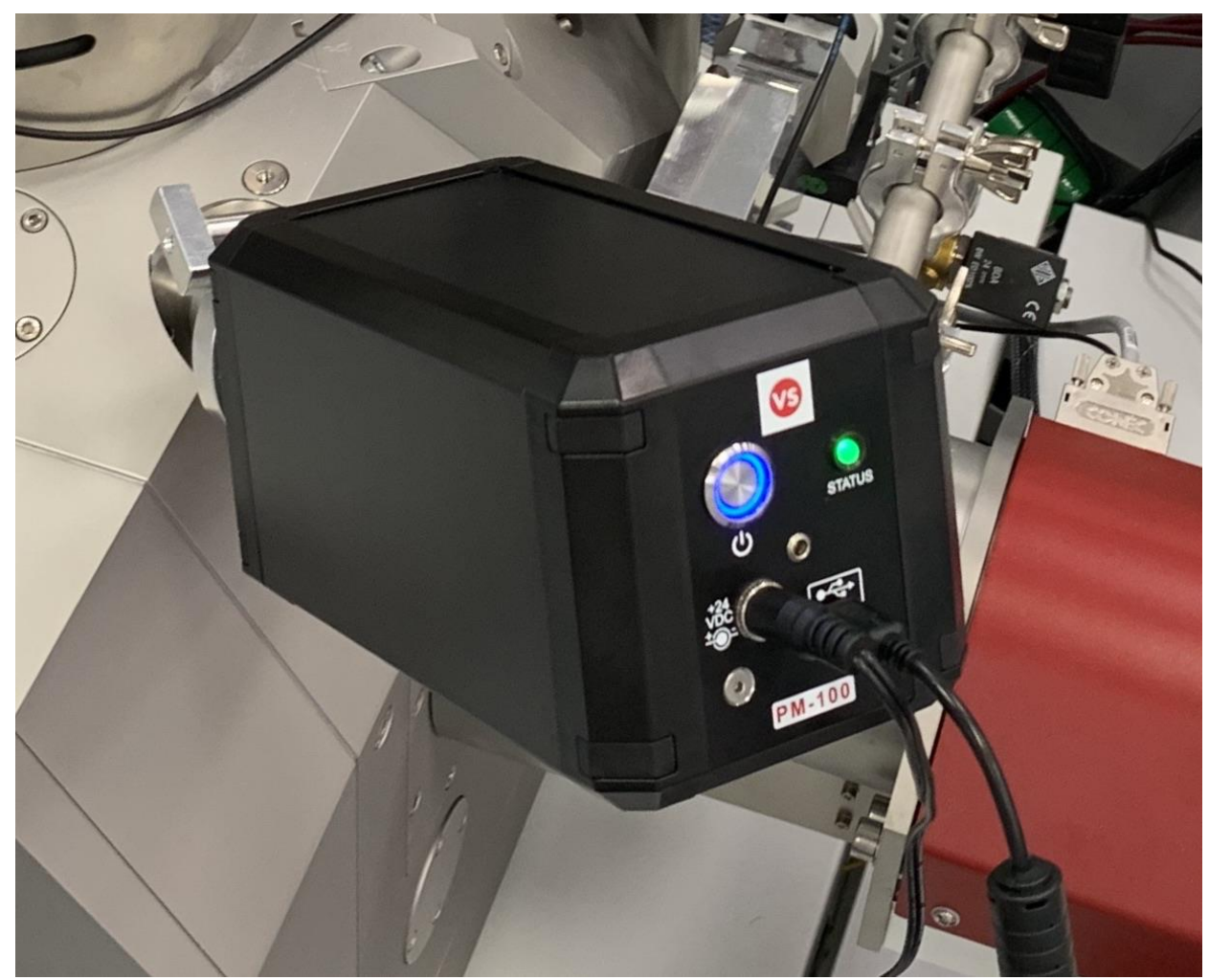

Figure 1. PM100 operating on SEM.

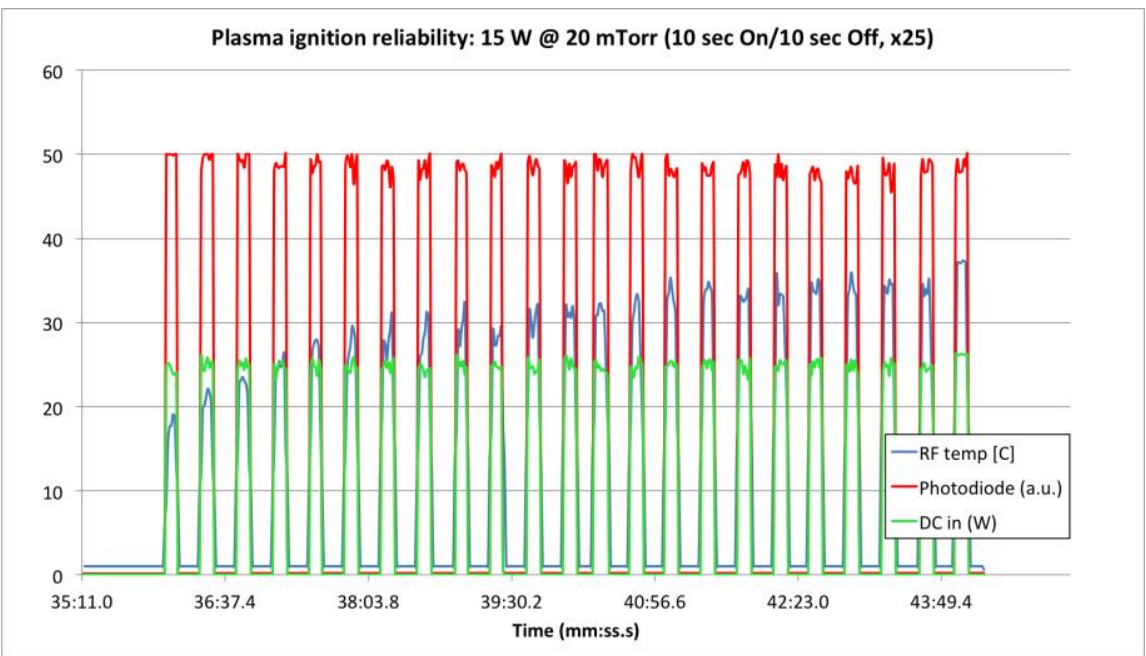

Figure 2. Ignition reliability over 25 cycles at 15 Watts power and 20 mTorr

References

1. C.A. Moore and G.J. Collins, M\&M Conference, Portland, OR (2019) 